\title{
Simple but effective: strong efficiency boost for a linear variable filter-based spectrometer
}

\author{
Aliaksei Kobylinskiy $\odot,{ }^{\text {a,b, } *}$ Matthias Kraus $\odot,{ }^{\text {a,b }}$ Xavier Uwurukundo, ${ }^{\text {a }}$ \\ Hartmut Hillmer, ${ }^{b}$ and Robert Brunner ${ }^{\mathrm{a}, \mathrm{c}}$ \\ ${ }^{a}$ University of Applied Sciences Jena, Applied Optics, Department SciTec, Jena, Germany \\ ${ }^{b}$ University of Kassel, Institute of Nanostructure Technologies and Analytics, Center for \\ Interdisciplinary Nanostructure Science and Technology, Kassel, Germany \\ ${ }^{c}$ Fraunhofer Institute for Applied Optics and Precision Engineering, Jena, Germany
}

\begin{abstract}
A method to drastically enhance detection efficiency of a linear variable filter (LVF) sensor across an extended and continuous wavelength range is presented. The efficiency is increased by a wavelength preselection concept, where the incoming light is divided into partial spectra to reduce otherwise unavoidable reflection losses of filter-based spectrometers. The simple but effective setup uses selected and successively arranged dichroic beamsplitters, which ensures an optimized compromise between efficiency enhancement and minimum increasing complexity. When connected to a two-dimensional camera and combined with a tilted LVF, this compact optical system allows the continuous recording of the full wavelength range between 450 and $850 \mathrm{~nm}$ with a resolution of $\sim 19 \mathrm{~nm}$ at $508.6 \mathrm{~nm}$. An efficiency enhancement factor of up to 5.7 is achieved in comparison to a conventional LVF setup. The working principle was verified by measuring the reflection spectra of different natural and artificial green leaves. The proposed approach for increasing the efficiency can be miniaturized and applied to a broad range of other filter-based sensors. (c) The Authors. Published by SPIE under a Creative Commons Attribution 4.0 International License. Distribution or reproduction of this work in whole or in part requires full attribution of the original publication, including its DOI. [DOI: 10.1117/1.JOM.1.4.044501]
\end{abstract}

Keywords: filter-based spectrometer; linear variable filter; increased detection efficiency; wavelength preselection.

Paper 21013 received Jun. 28, 2021; accepted for publication Sep. 3, 2021; published online Sep. 21, 2021.

\section{Introduction}

Spectroscopic analysis is no longer confined to science and laboratories. There is a growing demand for spectroscopic instruments with very ambitious instrumental requirements to work in extended application fields: process control for manufacturing industries, such as food ${ }^{1}$ and pharmaceutical ${ }^{2}$ industries, and screening a broad range of factors for agriculture and the environment, ${ }^{3}$ to name only a few. During the past decade, filter-based spectrometers have drawn attention, especially because of their potential for miniaturization. Filter-based spectrometers can have an extremely compact and rigid design without any moving parts, consisting of only a filter array or longitudinally variable filter placed in front of a sensor array, for example, a chargecoupled device (CCD) or complementary metal-oxide-semiconductor, to capture a spectrum in one shot. A variety of spectral filters and structures, often micrometer-sized, have been developed, such as plasmonic color filters, ${ }^{4,5}$ photonic crystal arrays ${ }^{6}{ }^{6}$ single nanowires, ${ }^{7}$ absorptive filter arrays composed of either conventional colloidal quantum $\operatorname{dots}^{8}$ or perovskite quantum dots, ${ }^{9}$ Fabry-Pérot (FP) filter arrays, ${ }^{10-12}$ and linear (also called "continuously") variable filters $^{13-22}$ [linear variable filters (LVFs)]. An important benefit of such types of spectrometers compared to grating-based spectrometers is the possibility of detecting a broad spectral range with high spectral resolution despite their small size. ${ }^{12}$ They can be implemented using a large number of narrowband filters or an appropriate gradient for the LVF. For example, the reported three FP filter arrays comprise 192 individual filters with a size of $40 \times 40 \mu \mathrm{m}$ and cover a

*Address all correspondence to Aliaksei Kobylinskiy, Aliaksei.Kobylinskiy@eah-jena.de 
spectral range between 521 and $685 \mathrm{~nm}$. The full width at half maximum (FWHM) of the individual transmission curves is nearly $3 \mathrm{~nm}$ (ranges from 1.7 to $5 \mathrm{~nm}$ ). ${ }^{11}$

Among the several advantages of filter-based spectrometers, a fundamental disadvantage is its low detection efficiency. This limited efficiency can be attributed to most of the broadband incident light passing through narrowband filters (or small regions of LVFs) being reflected or absorbed. Thus, even if ideally, the entire amount of light to be analyzed is distributed exactly over the filter array area (or along the dispersive axis of the LVF), most of the light fails to reach the detector. One approach to improve the efficiency of such filter-based spectrometers utilizes a spectral preselection method, in which the incoming light to be analyzed is divided spectrally and spatially into partial ray bundles and delivered to appropriate filter areas, which only cover a limited spectral range. A suitable implementation of the spectral preselection method requires a compromise between efficiency enhancement and minimum increasing complexity. There are various methods to accomplish a spectral preselection, e.g., using Köster prisms or micro prisms. Another simple approach uses multiple dichroic long-pass beamsplitters successively arranged and aligned to the filter arrays for the respective spectral subbands. Although the possibility of increasing efficiency was principally demonstrated earlier, ${ }^{23}$ this study was limited to a proof-ofconcept, restricted to a small 160-nm wide wavelength range and demonstrated without any spectrum acquisition. Experiments were based merely on intensity comparison and without losses analysis of the system.

The scope of this study refers for the first time to the application of the preselection concept to acquire a continuous spectrum across an extended wavelength range (450 to $850 \mathrm{~nm}$ ) without "wavelength gaps," thereby increasing the detection efficiency by a factor of up to nearly 6 . Therefore, a simple but effective approach of the preselection concept based on successively arranged dichroic beamsplitters was significantly expanded, combined with a tilted LVF, and connected to a 2D camera. Several broadband spectra were detected to demonstrate the working principle. In particular, the emission spectrum of a halogen lamp and the reflection spectra of natural and artificial leaves were measured. The transmission and reflection data of the beamsplitters and mirrors were used to estimate the module losses or approximate the increase in efficiency achieved.

\section{Working Principle}

In this work, the wavelength preselection principle was applied to create an efficiency-enhanced module employing an LVF as the spectral-resolving element. An LVF is a wedge filter that continuously changes the transmission wavelengths along one axis (longitudinal) while transmitting the same wavelengths along the perpendicularly oriented lateral axis. To date, several types of LVF have been developed, including metal-dielectric ${ }^{13}$ and all-dielectric structures. ${ }^{14-22}$ The latter ones became more prevalent and are already commercially available ${ }^{16,17}$ with wavelength ranges covering, e.g., 320 to 560,450 to 850 , and 800 to $1100 \mathrm{~nm},{ }^{16}$ or infrared (IR) regions, such as 0.9 to 1.70 or 2.5 to $5.0 \mu \mathrm{m} .{ }^{17}$ Numerous applications and devices based on LVFs have been reported, such as gas ${ }^{19}$ and liquid ${ }^{20}$ measurements, thin-film measurements, ${ }^{21}$ broadband spectrometers for current NASA space missions, ${ }^{22}$ order sorting filters for commercial grating spectrometers, ${ }^{24}$ multispectral and hyperspectral imaging, ${ }^{25,26}$ commercially available LVF monochromators ${ }^{27}$ and hyperspectral video cameras. ${ }^{28}$ The variable design of an LVF with different transmission distributions enables a flexible design of this concept.

Figure 1(a) shows the schematic of the LVF-based module. A broadband silver mirror redirects the incoming light (white light) to successively arranged dichroic long-pass beamsplitters (e.g., 1 to 5). These beamsplitters divide the incoming broadband beam into partial spectra or subbands (e.g., blue, green, and yellow subbands), which are reflected to the LVF and illuminate its entire longitudinal direction. Depending on the specific arrangement, this principle is multiple times more efficient than the conventional approach in which the white input light bundle is expanded to illuminate the entire LVF dispersive direction, as was realized, for example, in Ref. 20. Figure 1(b) shows an example using nine subbands by applying a matrix combining $3 \times 3$ beamsplitters to illuminate filter arrays. The matrix arrangement is beneficial concerning reduced cumulative transmission losses (see Sec. 4.4 of the study ${ }^{29}$ and Sec. 5 of this work). For 


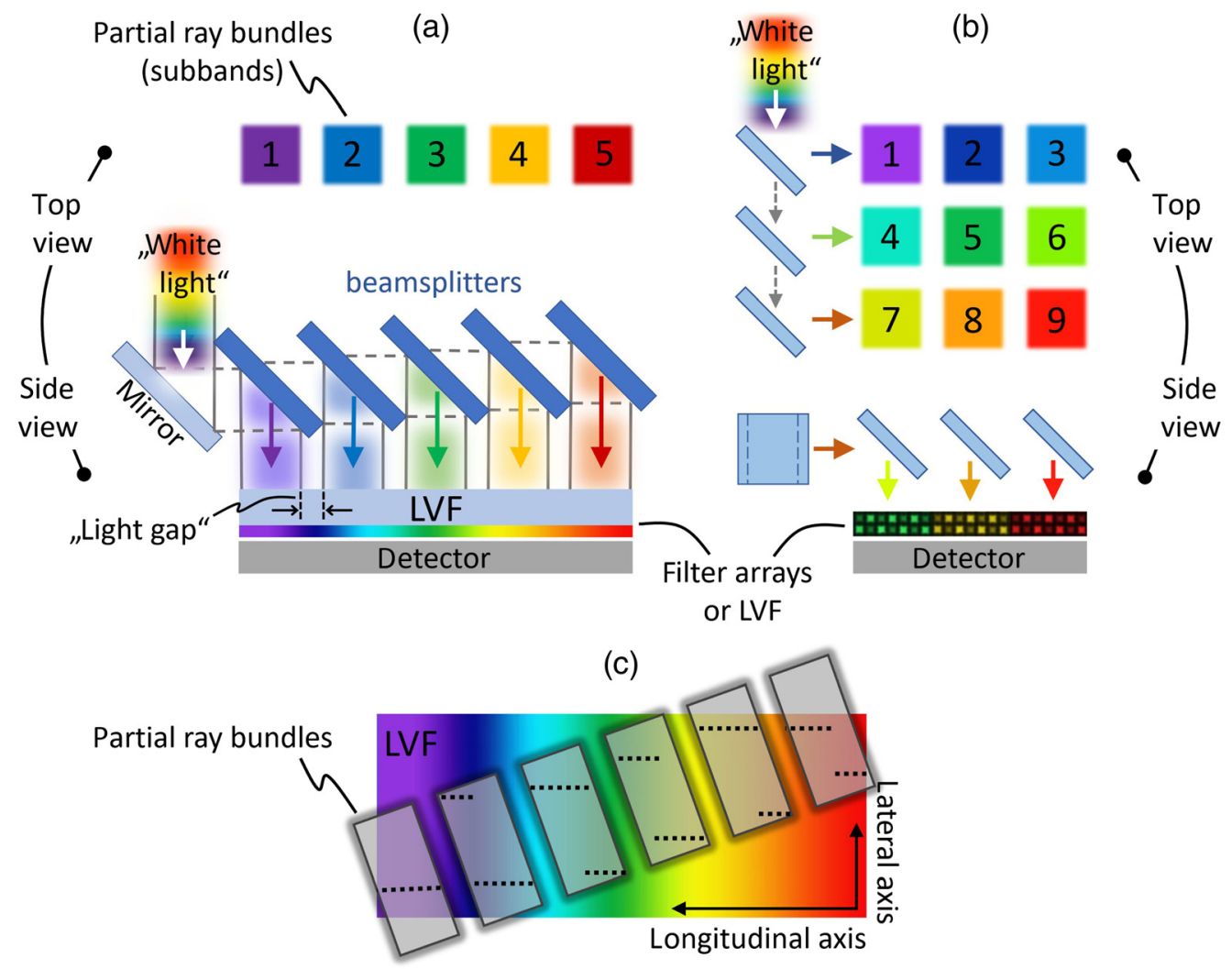

Fig. 1 Possible spectral preselection concepts based on dichroic beamsplitters. (a) The LVF is illuminated along its entire longitudinal direction with multiple ray bundles (e.g., 1 to 5 ), which are partial bands of the broadband incoming light divided by successively arranged dichroic long-pass beamsplitters (one row). The light distribution transmitted through the LVF hits the detector. (b) Example for 2D preselection: Nine subbands are created using a matrix of successively arranged beamsplitters to illuminate filter array groups (c) Method of continuous spectrum detection using rectangular ray bundles with their axes rotated relative to the LVF axes.

example, the last subband (number 9) in Fig. 1(b) is created by four transmissions and two reflections, instead of eight transmissions and one reflection, which are necessary when the preselection filters are arranged successively in a single row. In this study, using an LVF (transmission wavelength changes in one direction) the appropriate layout uses a row of beamsplitters, as shown in Fig. 1(a).

This simple layout possesses some problems that must be solved. First, the maximum number of dichroic elements is limited by the LVF size, substrate thickness of the dichroic elements, and cumulative transmission losses, which increase after transmission through each subsequent beamsplitter. Second, the thickness of the dichroic elements creates an unavoidable light gap on the LVF, even when the beamsplitters are positioned closest to each other. Thus, the substrate edge of the previous beamsplitter limits each bundle, as shown in Fig. 1(a), and an incomplete spectrum, where some wavelengths are lost, is obtained. The size of the gap can be estimated by knowing the thickness of the substrate and its refractive index. To reach the minimum possible gap, the incoming ray bundle should reflect from the deflection mirror in such a way that it passes the first beamsplitter (defined by refraction) close to the corner [see Fig. 1(a)]. By choosing the right distance between the beamsplitters, the optimal behavior of all ray bundles can be reached. The gaps may be compensated by introducing a slight tilt between the main axis of the LVF and the orientation of the dichroic filter stack, as shown in Fig. 1(c). A continuous spectrum without any wavelength gap can be generated by connecting the partial spectra represented by the dotted lines. Such an assembly is robust and easy to accomplish. 


\section{Mechanical Layout}

The mechanical layout of the spectrometer was based on selected commercially available optical elements. The LVF (LF103252; Delta Optical Thin Film ${ }^{16}$ ) offers the following relevant parameters: wavelength variation between 450 to $850 \mathrm{~nm}$ within a length of $24.7 \mathrm{~mm}, \mathrm{FWHM}$ of $4 \%$ at the central wavelength, and average transmission between $70 \%$ (blue region) and $90 \%$ (near-IR or NIR region). The blocking level was OD4 for a wavelength range of 200 to $1100 \mathrm{~nm}$. The substrate was $2 \mathrm{~mm}$ thick. Based on the physical and optical properties of the LVF, seven mirrors and beamsplitters were chosen to separate the incoming light into six spectrally and spatially separated ray bundles. The first and last were broadband reflective mirrors (Chroma Technology, sputtered enhanced silver mirror) with an average and absolute reflection of $\geq 98 \%$ and $>95 \%$, respectively, in the wavelength range of 380 to $1100 \mathrm{~nm}$. Figure 1(a) shows that the first mirror redirects the entire input light to the dichroic elements, whereas the last mirror reflects the last ray bundle, which is already transmitted through all beamsplitters, to the LVF. The other five optical elements (second to sixth) are dichroic long-pass beamsplitters, which operate at an angle of incidence of $45 \mathrm{deg}$. The choice of these beamsplitters is based on their availability provided by advanced manufacturers (Semrock, Chroma Technology) and their optical parameters are summarized in Table 1. Such beamsplitters are most commonly used in fluorescence spectroscopy (microscopy) and Raman spectroscopy. In combination with other (non-dichroic) filters, which offer better blocking factors of up to OD6, the dichroic beamsplitters are able to effectively separate an extremely weak signal from the intense excitation wavelength.

Figures 2(a)-2(d) illustrate the chosen beamsplitters with their transmission characteristics in connection with the LVF transmission data at certain positions and the applied geometry of the mechanics. The dimensions of the entrance aperture were chosen as $3.1 \times 10 \mathrm{~mm}$ to illuminate the entire longitudinal direction of the LVF. Thus, the six partial ray bundles are shown in Fig. 2(b) as bright rotated rectangles with dark edges. All consecutive optical elements were either 1 or $1.1 \mathrm{~mm}( \pm 0.05 \mathrm{~mm})$ thick, creating a minimal gap on the LVF surface between the partial ray bundles of nearly $1.2 \mathrm{~mm}$. The approach of continuous spectrum reading, which compensates for the gaps, is demonstrated by three colored lines in Figs. 2(b) and 2(d), and described in the next paragraph. This approach can be realized owing to the tilt position of the beamsplitter assembly relative to the LVF. The minimal angle of rotation $\alpha$ is defined as $\alpha=\arctan (g / w)$, where $g$ and $w$ are the gap width and length of the ray bundle (aperture), respectively. For this system, $\alpha=6.8 \mathrm{deg}$. A larger rotation of $24 \mathrm{deg}$ was applied to ensure a sufficient overlap of the previous and next ray bundles.

Figure 2(b) shows the black dashed lines on the LVF, dividing it into six equal sections, which are equal to the number of partial ray bundles for the system. These dashed lines define the longitudinal positions on the LVF, marked with different geometric symbols. The central transmission wavelengths of the LVF at these positions are shown in Fig. 2(c) based on the LVF transmission data. The five beamsplitters were chosen based on their edge wavelength (the position where the transmission is $50 \%$ ) with the closest possible value to the marked LVF values. The chosen beamsplitters satisfy the requirement almost exactly [compare the indicated values in Figs. 2(a) and 2(c)].

The quality of the continuous spectrum to be obtained depends not only on the selected optical elements but also on the appropriate pattern of the raw spectrum reading. The method is illustrated in Figs. 2(b) and 2(d) and demonstrates the reading of a part of the entire spectrum. First, the three yellow lines shown in Fig. 2(b) should be considered. The first line starts at the blue region of the LVF $(450 \mathrm{~nm})$ and ends optionally, where the second line starts at the next longitudinal pixel. At the position of the dashed line, the end of the second and the beginning of the third line accomplish the important transition between different partial ray bundles. Approximating this position intensity decreases to $50 \%$ because the dashed line position (at $520 \mathrm{~nm}$ ) matches the edge wavelength of the first beamsplitter [see Fig. 2(a)]. Thus, ideally, the expected raw spectrum measured by this method can possess only five wavelength regions with decreased intensity (near the five dashed lines). The spectral width of these regions where the intensity decreased is defined by the full edge of the beamsplitters, i.e., the spectral region between the end of the reflection and the beginning of the transmission band. For example, Table 1 shows that the entire edge of the first beamsplitter is 512 to $528 \mathrm{~nm}$. This reading method 

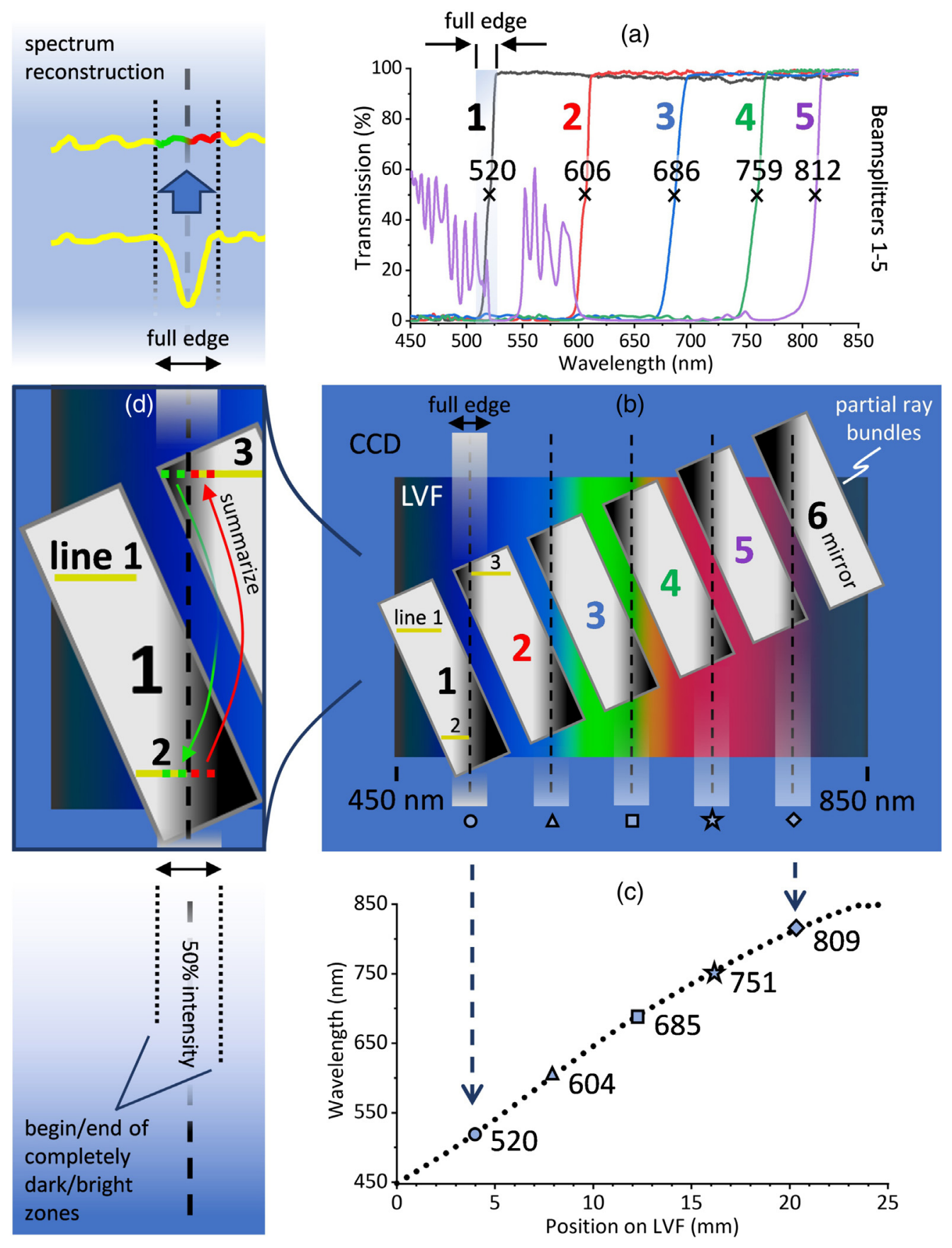

Fig. 2 Correspondence of the relative mechanical positions of the LVF with dichroic beamsplitters (1 to 5) and mirror (6) with their transmission curves. (a) Measured transmission curves of the beamsplitters from representative production lots with indicated edge wavelengths. (b) Representation of the mechanical layout between the LVF and partial ray bundles created using beamsplitters and mirrors. The dashed lines denote the lateral positions where the transmission peak of the LVF is observed. The yellow lines (1 to 3) present an approach of continuous spectrum reading. (c) Peak transmission wavelengths at different longitudinal positions of the LVF. (d) Prolonged yellow lines improve the approach of continuous spectrum reading.

is sufficient to create a continuous final spectrum from the raw data. However, for the cases in which the intensities of the measured spectrum approach zero near these edge positions, a slight improvement in this method is beneficial. Figure 2(d) shows the extension of the second yellow line by the red dotted line. Within the full edge of the first beamsplitter, the light detected by the red dotted line matches the missing portion of the light of the third yellow line. This seems to be 
Table 1 Optical parameters of the dichroic long-pass beamsplitters.

\begin{tabular}{lccccc}
\hline \hline $\begin{array}{l}\text { Beamsplitter } \\
\text { number }\end{array}$ & Part number & $\begin{array}{c}\text { Reflection } \\
\text { band } \\
(\mathrm{nm})\end{array}$ & $\begin{array}{c}\text { Transmission } \\
\text { band }^{\mathrm{n}}(\mathrm{nm})\end{array}$ & $\begin{array}{c}\text { Edge } \\
\text { wavelength } \\
\text { (nm) }\end{array}$ & $\begin{array}{c}\text { Angle of } \\
\text { incidence } \\
\text { (deg) }\end{array}$ \\
\hline 1 & FF520-Di02-25 $\times 36$ & 350 to 512 & 528 to 950 & 520 & $45 \pm 1.5$ \\
2 & FF605-Di02-25 $\times 36$ & 350 to 596 & 612 to 950 & 606 & $45 \pm 1.5$ \\
3 & FF685-Di02-25 $\times 36$ & 350 to 676 & 695 to 950 & 686 & $45 \pm 1.5$ \\
4 & FF757-Di01-25 $\times 36$ & 450 to 746 & 768 to 1100 & 759 & $45 \pm 1.5$ \\
5 & T810lpxr & 606 to 791 & 817 to 1179 & 812 & $45 \pm 1.5$ \\
\hline \hline
\end{tabular}

aSpecified as a spectral region with an average reflection $R_{\text {avg }}>98 \%$.

bSpecified as a spectral region with an average transmission $T_{\text {avg }}>93 \%$.

'Based on the manufacturers' measurements of representative production lots.

counterintuitive, but the values with the same longitudinal position of the red line should be sum up with the values of the third yellow line. More precisely, starting at the black dashed line position, third yellow and red lines have nearly the same intensity, which means an equal amount of light, 50\%, is reflected and transmitted by the first beamsplitter (and reflected by the second). Then, approaching the boarder of the indicated full edge, the end of the red line gets completely dark and the yellow line has the complete intensity. Thus, summing up the line values located at the same longitudinal LVF positions, the whole redistributed light is detected. The same procedure should be followed for the green dotted line shown in Fig. 2(d), which completes the intensity of the second yellow line. Finally, the entire edge region is reconstructed.
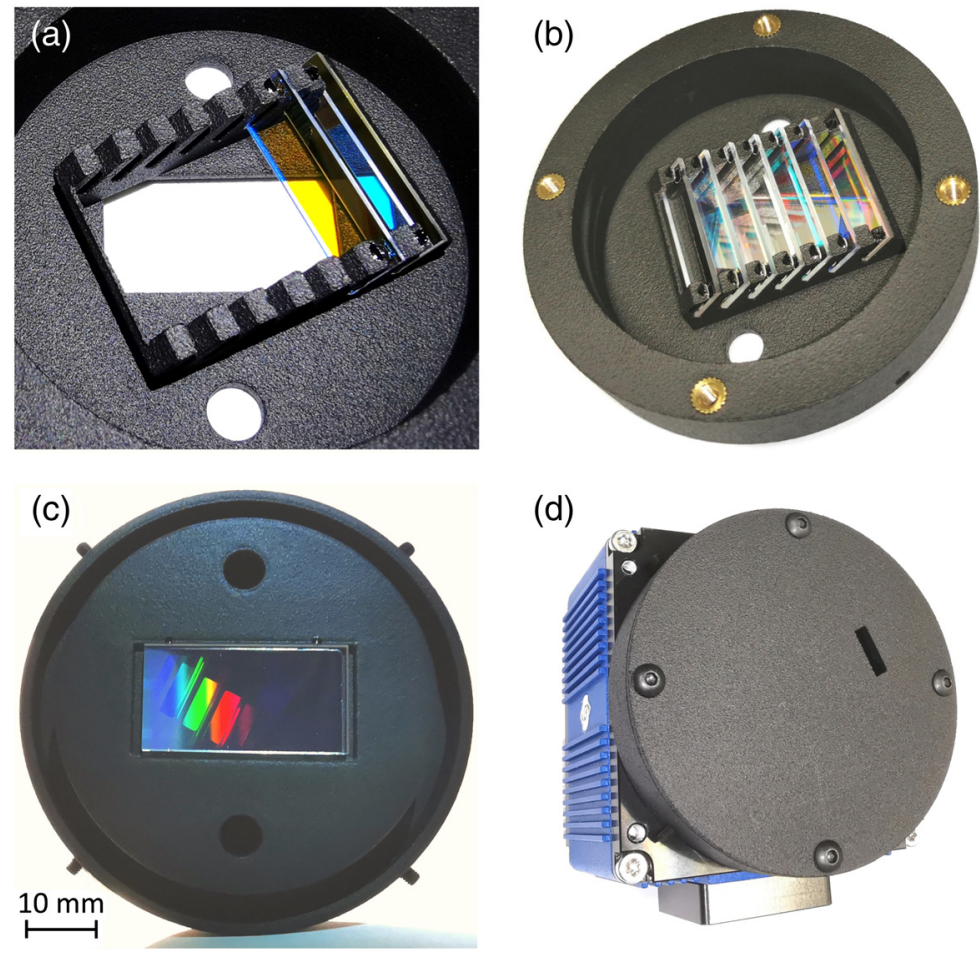

Fig. 3 Photographs of the optomechanical module. (a) Front side of the incomplete module, showing the relative angle between the LVF and dichroic beamsplitters/reflecting mirrors to be assembled. (b) Front side of the assembled module. (c) Rear side of the module, showing the LVF. As an example, some visible partial ray bundles appear when the closed module is pointed toward a halogen lamp. (d) Closed module connected to a CCD camera. A rectangular entrance aperture, $3.1 \times 10 \mathrm{~mm}$ in size, is visible. 
The chosen optical elements were mounted on an optomechanical module to connect to a CCD camera. Photographs of the module are shown in Figs. 3(a)-3(d). The housing was fabricated by a selective laser sintering method with black polyamide (PA12) as the applied material. Figure 3(a) shows the incomplete module with only the assembled first mirror and beamsplitter, where a relative angle of 24 deg between the LVF and beamsplitters to be mounted can be identified. The front side of the opened module with assembled mirrors, beamsplitters, and the LVF is shown in Fig. 3(b). Figure 3(c) shows the rear side of the closed module with the mounted LVF. In this photograph, the closed module was pointed toward a halogen lamp, making several partial ray bundles visible. The closed module connected to a CCD camera is shown in Fig. 3(d), where the rectangular entrance aperture is visible. The monochrome camera has a full-frame CCD sensor chip KAI-29050-A with $6576 \times 4384$ pixels and lateral pixel dimensions of $5.5 \times 5.5 \mu \mathrm{m}$. Owing to the protection glass of the CCD chip, the distance between the LVF and CCD chip is $1.7 \mathrm{~mm}$. To achieve better resolution, one side of the LVF with the narrow band layer stack faces the CCD chip, whereas the other side, the so-called "blocking side" of the LVF, faces the incoming light. A description of similar LVF layer structures can be found elsewhere. ${ }^{15}$

\section{Experimental Verification}

The continuous spectra should be analyzed to experimentally verify the working principle of the module. The module provides efficiency enhancement in the case where possibly all the available light to be analyzed is directed to the aperture. For example, this occurs when the light to be analyzed is delivered through a fiber with collimating optics. Thus, ideally, the parallel light bundle with a size approaching that of the aperture enters the system and then gets spatially and spectrally distributed to illuminate the entire LVF. Based on this principle, the following measurements were obtained using an optical fiber: the emission spectra of a halogen lamp and mercury-cadmium ( $\mathrm{HgCd}$ ) spectral lamp, used mainly for calibration, and the reflection spectra of artificial and natural leaves.

For all measurements, a multimode optical fiber with a core diameter of $105 \mu \mathrm{m}$ and an NA of 0.1 was used (Thorlabs M94L02). To focus and collimate the light to be analyzed, two protected silver reflective collimators were used (Thorlabs RC08FC-P01 and RC12FC-P01). Their average reflectance was $>97.5 \%$ for a wavelength range of 0.45 to $2 \mu \mathrm{m}$, and they were based on off-axis parabolic mirrors. The RC12FC-P01 collimator was used to illuminate the entrance aperture (another one, RC08FC-P01, to focus the light into a fiber). Using this collimator with effective focal length of $50.8 \mathrm{~mm}$ in conjunction with the $0.1 \mathrm{NA}$ optical fiber, a collimated output beam with a diameter of $\sim 10.16 \mathrm{~mm}$ was created.

The assembled system was adjusted by illuminating with a halogen lamp. Figures 4(a)-4(d) show the measurement results of the halogen lamp using the spectrum reading method and its improved modification (see the penultimate paragraph of the third section). The primary method, without improvement, is crucial for understanding the spectra to be presented and is also used in the last section. To recognize the initial spectrum of the source, the normalized spectrum of the same halogen lamp generated by the commercial JETI spectroradiometer (Specbos 1211UV) is presented in Fig. 4(b) as a red dashed line. After choosing the appropriate reading line pattern, shown by the colored longitudinal lines in Fig. 4(a) and in Fig. 4(c), five pixel rows were averaged.

For simplicity, all spectra are already calibrated, and the horizontal axes are shown in wavelengths. The LVF transmission data presented in Fig. 2(c) and the raw spectrum of the $\mathrm{HgCd}$ spectral lamp were used for calibration, i.e., convert the spatial pixel intensity information (gray value for pixel number) into pixel intensity as a function of incident wavelength. The CCD camera image and raw spectrum of the HgCd lamp are shown in Figs. 4(e) and 4(f), respectively. For the calibration, two detected spectral lines of the $\mathrm{HgCd}$ lamp, 643.9 and $508.6 \mathrm{~nm}$, were used, and the tabulated values were precisely defined using the atomic spectra database. ${ }^{30}$ To ensure correct calibration, some other detected spectral lines, whose tabulated values were $546.1 \mathrm{~nm}$ and double line 577 and $579.1 \mathrm{~nm}$, were found at 547.6 and $577 \mathrm{~nm}$ (double line), respectively. 

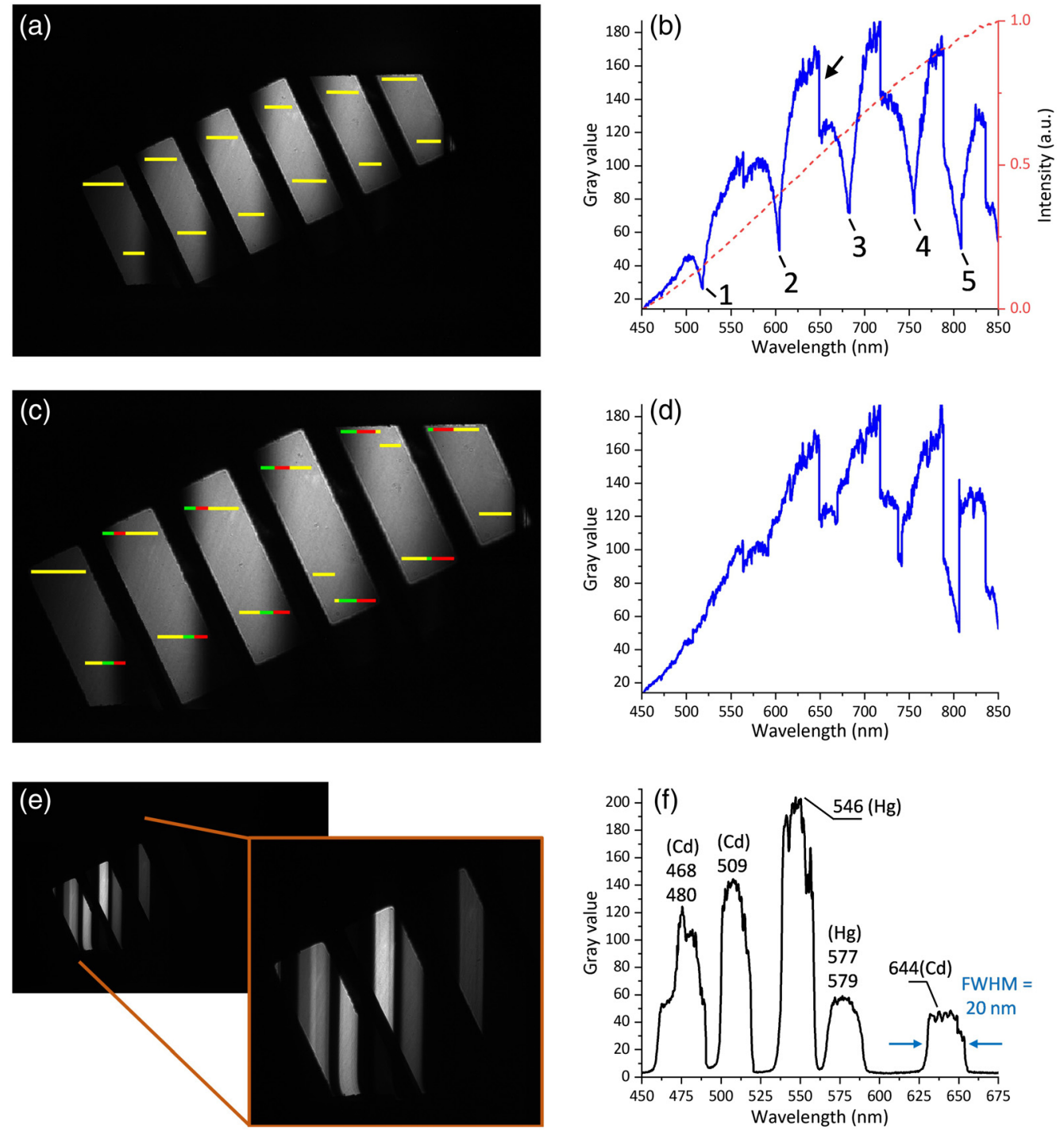

Fig. 4 Measurements of continuous spectra. (a) CCD camera image when measuring the spectrum of the halogen lamp. The yellow lines indicate the measurement directions. (b) Raw spectrum of the halogen lamp (blue curve) and the reference halogen lamp spectrum measured using a commercial spectroradiometer (dashed red curve). (c) Improved reading method applied to the CCD image of the halogen lamp. The rearranged yellow lines, in connection with red and green lines, complete the previously decreased regions of the raw spectrum. (d) Reconstructed raw spectrum of the halogen lamp using an improved reading method (e) CCD camera image when measuring the spectrum of the HgCd lamp and its magnified active part. (f) Raw spectrum of the HgCd lamp.

The raw spectrum of the $\mathrm{HgCd}$ lamp also demonstrated the resolution of the system. The FWHM of the fitted curves was nearly $4 \%$ of the central wavelength, close to the specification value of the LVF. Figure 4(f) shows that the obtained FWHM of the spectral lines increases toward longer wavelengths. For example, the FWHM of the 508.6 and $643 \mathrm{~nm}$ lines were nearly 18.7 and $20 \mathrm{~nm}$, respectively. Two lines, 467.8 and $480 \mathrm{~nm}$, were not resolved.

Next, we present a more detailed discussion of the raw spectra of the halogen lamp shown in Figs. 4(b) and 4(d) measured using the primary and improved reading approaches, respectively. The raw spectra differ strongly from the reference spectrum measured using a commercial spectroradiometer, shown by the red dashed line in Fig. 4(b). This can be explained on the basis of several factors that influence the initial spectrum: CCD sensor response and its cover glass transmission, transmission of the LVF at different longitudinal positions, a combination of 
transmission and reflection data of dichroic elements for each of the six partial ray bundles, and intensity distribution of the collimated beam. The influence of this beam intensity distribution is clearly visible as abrupt decreases in the spectrum, which appear because of the spectrum reading method-different reading lines are relocated over the partial ray bundles with specific intensity distributions. Thus, sudden changes in the intensity level appeared accordingly. One such decrease is indicated by the black arrow in Fig. 4(b). Other features, such as relatively fast but continuous decreases, are present in the spectrum generated using the primary reading method. This generated raw spectrum contains five regions with decreased intensities, indicated by the numbers 1 to 5 in Fig. 4(b). These correspond to the regions where the edge wavelengths of the beamsplitters appear. The lowest positions of these decreased regions (1 to 5) occur at 518, 605, 685, 757, and $809 \mathrm{~nm}$. This proves the correct optomechanical layout presented in Fig. 2 and wavelength calibration. To reconstruct these decreased regions, the improvement in the reading method was applied. As explained in Fig. 2(d), extending the yellow lines by red and green lines and summing up the corresponding values produces the "repaired" raw spectrum shown in Fig. 4(d). This is accomplished by rearranging the yellow lines to extend the additional red and green lines as much as possible. It was possible to fully reconstruct the decreased regions from one to four (see also the previously mentioned influence of the beam intensity distribution), but not the fifth region, owing to the nonlinearity of the LVF transmission data in connection with the symmetrical mechanical layout. Nevertheless, the raw spectra have a sufficient gray value level along the entire detection region that avoids loss of information during continuous spectrum acquisition. The shown improvement in the reading method is beneficial for spectra with a strong intensity amplitude approaching zero within the spectrum.

To provide application-oriented measurements, the reflection spectra of green natural and artificial leaves were obtained. The final spectra of these specimens were generated in three simple steps. The first two steps are the consecutive measurements of the source and reflection
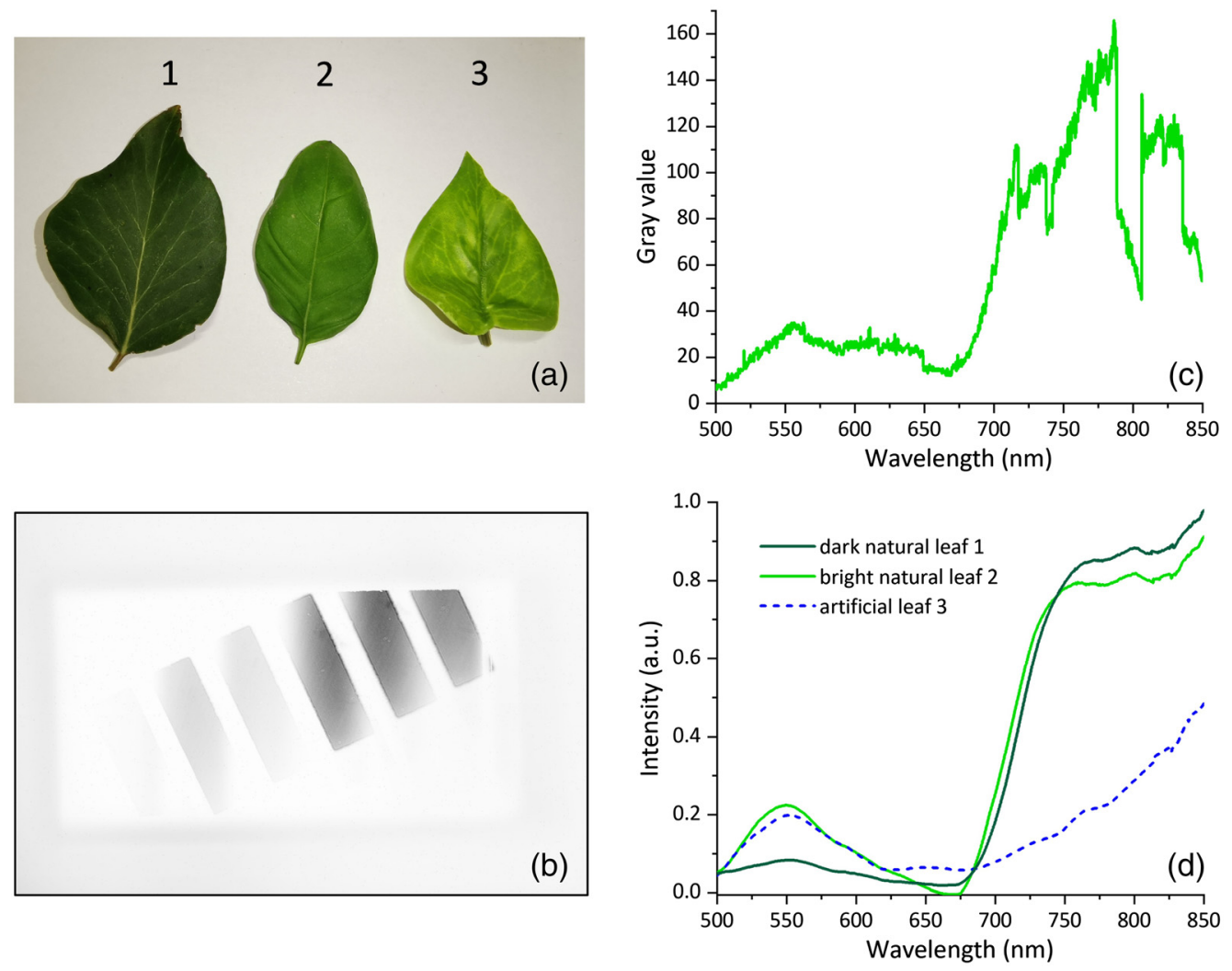

Fig. 5 (a) Photograph of the measured green natural leaves (leaf number one and two) and an artificial leaf (number three). (b) Negative CCD camera image during measurements of the reflection spectrum of the second natural green leaf illuminated by a halogen lamp. (c) Raw reflection spectrum of the second natural green leaf. (d) Calculated reflection spectra of the leaves. 
of the sample placed in the path of the beam. The third step is the division of the raw sample spectrum by the raw source spectrum, which delivers the desired final spectrum of the sample. A photograph of the measured leaves is shown in Fig. 5(a). The dark and bright natural leaves are indicated by numbers one and two, and artificial leaf is indicated by the number three. As an example, Fig. 5(b) shows an inverted CCD camera image obtained by measuring the second natural leaf illuminated by a focused halogen lamp. The raw reflection spectrum of this leaf is shown in Fig. 5(c). Several decreased intensity regions are explained by the same influences described in the previous two paragraphs and can also be observed in Fig. 4(d).

The final calculated reflection spectra of the leaves are shown in Fig. 5(d). The resulting spectra demonstrate the well-known behavior of green natural leaves: one clear peak at approximately $550 \mathrm{~nm}$ and a strong increase (known as the red-edge) between $\sim 680$ and $710 \mathrm{~nm}$ with a further high reflectance value in the NIR range owing to the chlorophyll content. In contrast, the artificial green leaves do not exhibit a clear increase in reflectance in the red and NIR wavelength regions. It is to be noted that the calculated spectra of the leaves start at $500 \mathrm{~nm}$ because of the weak signal between 450 and $500 \mathrm{~nm}$.

\section{Achieved Efficiency Increase and Spectra Simulations}

The proposed system is shown to be capable of measuring continuous spectra in the wavelength range of 450 to $850 \mathrm{~nm}$. To estimate the efficiency enhancement achieved, the intensities of the input light bundle of the presented and typical systems should be compared in conjunction with the cumulative transmission losses of the beamsplitters used. The comparison of the intensities of the light bundles is based on the comparison of the areas of the light bundles required to illuminate the presented and typical case of the system, because the intensity is inversely proportional to the illuminated area. By creating six partial ray bundles, the proposed system based on the optomechanical layout described earlier (see the first two paragraphs of the third section) achieves an efficiency increase factor of $\sim 6$, neglecting the losses of the beamsplitters.

The calculations for estimating the losses of the beamsplitters were based on their optical characteristics. The transmission data of all dichroic beamsplitters, which are shown in Fig. 2(a), and two mirrors were used to create the continuous graph shown in Fig. 6(a). This graph demonstrates the amount of light available after transmission and reflection during the spectral preselection process. The intensity values drop from 450 to $850 \mathrm{~nm}$ owing to the cumulative losses of the consecutive beamsplitters. To create this continuous graph, the entire spectral region was divided into eleven subregions $n$. These consist of six regular reflection regions of partial ray bundles and five edge regions (transition regions). For each subregion, the loss coefficient $C_{n}$ is defined. After interacting with the beamsplitters, the initial intensity $I_{0}(\lambda)$ changes to $I_{n}(\lambda)=C_{n} I_{0}(\lambda)$, where $I_{n}(\lambda)$ is the resulting intensity of the appropriate subregion $\mathrm{n}$. For the graph shown in Fig. 6(a), we assumed that $I_{0}=1$ for all wavelengths. Thus, the values of the loss coefficient $C_{n}$ over the entire 450 to $850 \mathrm{~nm}$ region can be obtained.

For each subregion, the appropriate combination of the reflection and transmission values of the beamsplitters and mirrors is defined as the loss coefficient $C_{n}$. Since absorption is assumed to be zero, the transmission and reflection values are described by the simple relation $R=1-T$. As an example, the light of the first partial ray bundle in the regular reflection region is created by the reflection from the first deflection mirror $\left(R_{m}\right)$ and the first beamsplitter $\left(R_{B 1}\right)$. Consequently, the loss coefficient for this 450 to $512 \mathrm{~nm}$ region is described as $R_{m} R_{B 1}$. The second region 512 to $528 \mathrm{~nm}$ is the transition region: full edge of the first beamsplitter. The loss coefficient of the second region is defined as $R_{m}\left(R_{B 1}+T_{B 1} R_{B 2}\right)$. Thus, the light after reflection on the deflection mirror $\left(R_{m}\right)$ is redistributed by the first beamsplitter (reflected and transmitted, $R_{B 1}$ and $T_{B 1}$, respectively), and the transmitted part is reflected by the second beamsplitter $R_{B 2}$. Continuing the procedure, all the loss coefficients can be defined. The maximum loss of the system is precisely defined by the last loss coefficient as $R_{m} T_{B 1} T_{B 2} T_{B 3} T_{B 4} T_{B 5} R_{m}$. This indicates that the light of the last region $(817$ to $850 \mathrm{~nm}$ ) is reflected by the first mirror, then transmitted through all five beamsplitters, and finally reflected by the last mirror. In the last wavelength region ( 817 to $850 \mathrm{~nm}$ ), an average value of nearly $85 \%$ of the initial intensity was achieved, as shown in Fig. 6(a). Curve oscillations originate from the multiplication of the 

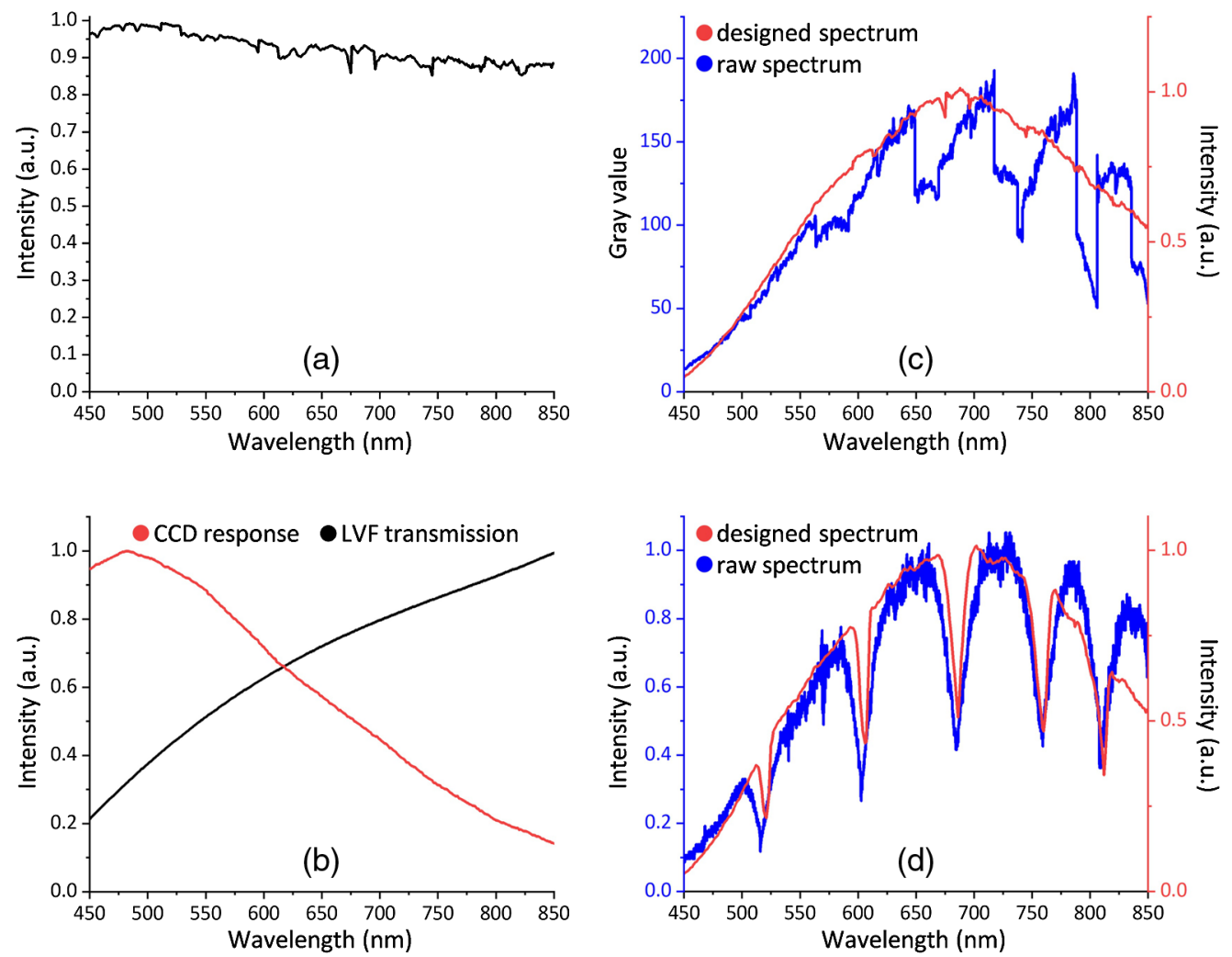

Fig. 6 (a) Cumulative transmission and reflection losses of the beamsplitters and mirrors. (b) CCD sensor response with its cover glass transmission (red curve), normalized curve generated from integrated transmission intensities of the LVF at different longitudinal positions (black curve). (c) Comparison of the measured raw spectrum of the halogen lamp (blue curve) and theoretical (designed) raw spectrum of the halogen lamp (red curve). The spectra are created with the improved spectrum reading method, as shown in Fig. 4(d). (d) Comparison of the measured raw spectrum (blue curve) of a halogen lamp (placed $3.5 \mathrm{~m}$ away) and the theoretical raw spectrum (red curve) of the halogen lamp. The spectra are evaluated with the primary reading method, shown earlier in Fig 4(b) for fiber-based measurements.

discrete transmission/reflection data of the beamsplitters. This includes oscillations arising from the dielectric interference coatings of the beamsplitters [see Fig 2(a)]. Considering the initially discussed efficiency enhancement factor of 6 and the 15\% losses, the final efficiency increase factor for the last region is $\sim 5.1$. For the previous regions, the factor is larger and is $\sim 5.7$ for the first region (450 to $512 \mathrm{~nm}$ ), where losses of up to $5 \%$ appear owing to reflections on the first deflection mirror and the first beamsplitter.

To support the correct estimation of the beamsplitters losses we simulated the raw CCD signal of the halogen lamp and compared it with the experimental raw spectrum shown in Fig. 4(d). The raw spectrum of the halogen lamp was simulated (predicted) by incorporating the factors influencing the system, i.e., the transmission and reflection characteristics of all the optical elements used and the CCD sensor response. Figure 6(a) shows the beamsplitters losses described earlier. Figure 6(b) shows the CCD sensor response with its cover glass transmission (red curve) and normalized curve generated from the integrated transmission intensities of the LVF at different longitudinal positions (black curve). These defined system influences were multiplied with the initial spectrum data of the halogen lamp, which was measured using the spectroradiometer shown in Fig. 4(b) and used as a reference herein. Figure 6(c) presents a comparison of the raw spectrum of the halogen lamp measured with a collimated optical fiber output (blue curve) and a simulated raw spectrum for the case of an ideally parallel input light (red curve). The two spectra presented in Fig. 6(c), theoretical and experimental, show quite similar enveloping behavior. The main differences can be explained by the lateral intensity profile of the input light bundle in connection with the spectrum reading method. In addition, close to the edge 
of the last beamsplitter, a strongly decreased region is observed at $\sim 810 \mathrm{~nm}$ (see the penultimate paragraph of the fourth section).

The validity of the theoretical predictions can be emphasized by another simple spectra comparison. Therefore, a measurement was conducted using a distant halogen lamp ( $\sim 3.5 \mathrm{~m}$ away). In this configuration, a quite parallel light bundle enters the system at the location of the entrance aperture. More precisely, with a light bulb filament nearly $10 \mathrm{~mm}$ in size, the full divergence of the source is only $2.8 \mathrm{mrad}(0.16 \mathrm{deg})$ at the position of the entrance aperture. Figure $6(\mathrm{~d})$ shows a comparison of the raw spectrum of the measured halogen lamp and the theoretically designed spectrum. Here, the primary reading method, shown earlier in Fig. 4(b) for fiber-based measurement, was used for this comparison to additionally demonstrate the positions of the edge wavelengths of the beamsplitters. The theoretical and experimental values shown in Fig. 6(d) are very similar, proving the well-designed theoretical reconstruction. The differences between two curves could be attributed to several factors. The main factors are probably the residual divergence of the source (distant halogen lamp) and residual stray light inside the housing. In connection with the long caption time it may be the reason for excessive intensity of the raw spectrum curve (especially IR region) in comparison to the designed curve. Some minor factors are uncertainties caused by calibration and reading processes and by adjusting the module. Consequently, based on the mentioned comparison, the estimated beamsplitters losses of a maximum of $15 \%$ and an efficiency increase factor of up to 5.7 are considered plausible.

\section{Conclusions}

An efficiency enhanced module for continuous spectra acquisition based on multiple beamsplitters and an LVF was presented, including its simple assembly, measurement method, and loss analysis. An efficiency increase factor of more than five was achieved with the chosen optomechanical layout. The working principle is demonstrated by the measurements of the emission spectrum of a halogen lamp and reflection spectra of natural and artificial green leaves-most importantly, without the loss of any wavelengths in the entire spectral region (450 to $850 \mathrm{~nm}$ ). This system was accomplished using only commercially available LVF and beamsplitters. Using freely designed LVF parameters, such as dimensions, wavelength range, and resolution, application-tailored efficient spectrometers can be designed. Other filter types, such as quantum dot systems, may be expected to also benefit from spectral preselection if an appropriate arrangement is implemented.

Furthermore, it was shown that beamsplitters already exhibit fully sufficient characteristics and produce reasonable results because of their moderate losses. Thus, we demonstrated the high potential of the spectral preselection concept for diverse filter types, as well as extended the established reasonable limit for using successively arranged beamsplitters for other reported applications, such as color imaging. ${ }^{29}$ The beamsplitters could also be customized to increase the edge steepness or increase the reflection and transmission even closer to $100 \%$ for the desired spectral region, for the most effective spectral preselection. It is believed that thin-film technology can aid the successful implementation of a simple concept in the near future.

Planned future work includes customized dichroic optics and improved arrangement of dichroic elements, which could be based on the increased use of reflection (or multiple reflections), along with the continuous search of filter types whose efficiency could be drastically increased. To improve the resolution of the system other filter type (or alternatively filter array) with better resolution should be taken. Finally, this spectral preselection concept may be applied to hyperspectral imaging systems, which are also limited by low efficiency. The solution can be based on creating an intermediate image and an extended parallel light bundle within such systems.

\section{Acknowledgments}

The authors thank the Carl Zeiss Spectroscopy GmbH, Germany, Scia Systems GmbH, Germany, and Bundesministerium für Bildung und Forschung (Grant No. 13FH657IX6) for financial support. Disclosures: The authors declare no conflicts of interest. 


\section{Code, Data, and Materials Availability}

Data underlying the results presented in this paper are not publicly available at this time but may be obtained from the authors upon reasonable request.

\section{References}

1. G. Downey Ed., Advances in Food Authenticity Testing, Woodhead Publishing (2016).

2. E. W. Ciurczak and B. Igne, Pharmaceutical and Medical Applications of Near-Infrared Spectroscopy, CRC Press (2014).

3. Z. Tümsavaş et al. "Prediction and mapping of soil clay and sand contents using visible and near-infrared spectroscopy," Biosyst. Eng. 177, 90-100 (2019).

4. T. $\mathrm{Xu}$ et al., "Plasmonic nanoresonators for high-resolution colour filtering and spectral imaging," Nat. Commun. 1, 59 (2010).

5. D. Fleischman et al., "Hyper-selective plasmonic color filters," Opt. Express 25(22), 2738627395 (2017).

6. K. M. Bryan et al., "Inexpensive photonic crystal spectrometer for colorimetric sensing applications," Opt. Express 21(4), 4411-4423 (2013).

7. Z. Yang et al., "Single-nanowire spectrometers," Science 365(6457), 1017-1020 (2019).

8. J. Bao and M. G. Bawendi, "A colloidal quantum dot spectrometer," Nature 523(7558), 67-70 (2015).

9. X. Zhu et al., "Broadband perovskite quantum dot spectrometer beyond human visual resolution," Light Sci. Appl. 9(1), 73 (2020).

10. Y. Horie et al., "Wide bandwidth and high resolution planar filter array based on DBR-metasurface-DBR structures," Opt. Express 24(11), 11677-11682 (2016).

11. Y. Shen et al., "Fabrication and characterization of multi-stopband Fabry-Pérot filter array for nanospectrometers in the VIS range using SCIL nanoimprint technology," Appl. Nanosci. 8(6), 1415-1425 (2018).

12. H. Hillmer et al., "Role of nanoimprint lithography for strongly miniaturized optical spectrometers," Nanomaterials 11, 164 (2021).

13. A. Piegari, J. Bulir, and A. K. Sytchkova, "Variable narrow-band transmission filters for spectrometry from space. 2. Fabrication process," Appl. Opt. 47(13), C151-C156 (2008).

14. A. Emadi et al., "Design and implementation of a sub-nm resolution microspectrometer based on a linear-variable optical filter," Opt. Express 20(1), 489-507 (2012).

15. H. Tang et al., "Preparation and spectrum characterization of a high quality linear variable filter," Coatings 8(9), 308 (2018).

16. https://www.deltaopticalthinfilm.com/linear-variable-filters

17. https://vortexopticalcoatings.co.uk/linear-variable-filters-2

18. A. Emadi et al., "Linear variable optical filter-based ultraviolet microspectrometer," Appl. Opt. 51(19), 4308-4315 (2012).

19. N. P. Ayerden, G. de Graaf, and R. F. Wolffenbuttel, "Compact gas cell integrated with a linear variable optical filter," Opt. Express 24(3), 2981-3002 (2016).

20. Y. Wan et al., "Compact characterization of liquid absorption and emission spectra using linear variable filters integrated with a CMOS imaging camera," Sci. Rep. 6(1), 29117 (2016).

21. P. J. Murr et al., "Thin film measurement system for moving objects based on a laterally distributed linear variable filter spectrometer," Rev. Sci. Instrum. 83(3), 035110 (2012).

22. K. Hendrix, "Linear variable filters for NASA's OVIRS instrument: pushing the envelope of blocking," Appl. Opt. 56(4), C201-C205 (2017).

23. A. Kobylinskiy et al., "Substantial increase in detection efficiency for filter array-based spectral sensors," Appl. Opt. 59(8), 2443-2451 (2020).

24. https://ibsen.com/products/oem-spectrometers/freedom-spectrometers

25. M. Hubold et al., "Multi-aperture system approach for snapshot multispectral imaging applications," Opt. Express 29, 7361-7378 (2021).

26. I. G. E. Renhorn et al., "High spatial resolution hyperspectral camera based on a linear variable filter," Opt. Eng. 55(11), 114105 (2016). 
27. https://www.bmglabtech.com/lvf-monochromators

28. https://cubert-gmbh.com/product/ultris-20-hyperspectral

29. N. Hagen and M. W. Kudenov, "Review of snapshot spectral imaging technologies," Opt. Eng. 52(9), 090901 (2013).

30. https://physics.nist.gov/PhysRefData/ASD/lines_form.html

Aliaksei Kobylinskiy received his diploma in physics in 2013 from the Belarussian State University, Minsk, Belarus, and his MEng degree in 2019 in the field of optical and laser technology from the University of Applied Sciences Jena, Germany. Currently, he is a PhD student at the University of Kassel and the University of Applied Sciences Jena, Germany. His research fields are micro optics and filter-based spectrometers.

Matthias Kraus received his BEng and MEng in the field of optical and laser technology from the University of Applied Sciences Jena, Germany, in 2011 and 2013, respectively. Since 2013, he has worked as a scientific assistant at the University of Applied Sciences Jena and since 2016 as a $\mathrm{PhD}$ student at the University of Kassel, Germany. His research fields are crossed diffraction gratings for spectroscopy, nanostructured anti-reflective coatings, optical lithography, and reactive ion beam etching.

Xavier Uwurukundo received his BSc degree in 2013 in the field of optical technology and image processing from the Darmstadt University of Applied Sciences, Germany. Currently, he is a MEng student and scientific assistant at the University of Applied Sciences Jena, Germany in the field of applied optics. His main interest is image processing.

Hartmut Hillmer received his $\mathrm{PhD}$ from Stuttgart University in 1989 on electronic transport in semiconductor QWs and his habilitation degree from TU Darmstadt in 1996 on the QW material system AlGaInAs. He worked at Research Center German Telekom from 1989 to 1999 on semiconductor lasers, and NTT Optoelectronic Laboratories, Japan, in 1991. He has been a professor since 1999 and a board member since 2016 of Graduate Academy at the University of Kassel. He received "European Grand Prix for Innovation Awards" in 2006. His research fields are microsystem technology, nanosensors and actuators for smart personal environments, and active windows.

Robert Brunner received his $\mathrm{PhD}$ in the field of near-field optical microscopy from the University of Ulm (Germany) in 1998. Between 1998 and 2009, he worked for the Research Center of Carl Zeiss where, he was the responsible lab manager for Microstructured Optics. Since 2010 he has been a professor for applied optics at the University of Applied Sciences in Jena. His current research interests are hybrid diffractive/refractive optics, subwavelength structures, refractive microoptics, lithography, and high resolution optics. 